\title{
Lympho-Vascular Space Invasion Indicates Advanced Disease for Uterine Papillary Serous Tumors Arising from Polyps
}

\author{
Selcuk Ilker ${ }^{*}$, Korkmaz Elmas ${ }^{1}$, Ozgu Emre $^{1}$, Turker Mengu ${ }^{2}$, Salim Erkaya $^{3}$, \\ Gungor Tayfun 4
}

\begin{abstract}
Background: Uterine papillary serous tumors are rarely seen and behave aggressively. Our aim was to evaluate uterine papillary serous tumors arising from polyps. Materials and Methods: Clinicopathological data of patients with uterine serous cancer arising from a polyp at the Gynecological Oncology Department of Zekai Tahir Burak Women's Health Education and Research Hospital were reviewed retrospectively. Results: We analyzed patients according to FIGO 2009 staging system as stage $1 \mathrm{~A}$ and higher than stage $1 \mathrm{~A}(3$ and 6 , respectively). All the patients were postmenopausal. Mean CA-125, CA-19.9 and CA15.3 levels were elevated in higher than stage $1 \mathrm{~A}$ group. However we did not find a statistical difference between age, parity, polyp size, CA-125, CA-15.3, CA-19.9 and CEA levels. Lympho-vascular space invasion (LVSI) showed predictivity for advanced disease $(\mathrm{p}=\mathbf{0 . 0 2 5})$. Conclusions: The histopathologic nature of uterine serous carcinoma is a unique entity. LVSI is a prognosticator for defining an advanced stage uterine papillary tumor.
\end{abstract}

Keywords: Uterine cancer - serous - LVSI - myometrial - polyp

Asian Pac J Cancer Prev, 16 (10), 4257-4260

\section{Introduction}

Endometrial cancer is the most common gynecologic malignancy (Siegel et al., 2013). Endometrioid histology is the most commonly detected type with a favorable prognosis. Although uterine papillary serous carcinoma (UPSC) is rarely seen; less than $10 \%$ of all uterine cancers (Moore and Fader, 2011), it behaves very aggressively and UPSC represents more than $50 \%$ of all endometrium cancer related deaths and recurrences (del Carmen et al., 2012). Even in completely debulked, node negative, stage 1 UPSC patients five-year overall survival is not more than $70 \%$ (Huh et al., 2003). Additionally approximately $60 \%$ of UPSC patients are diagnosed with greater than stage 1 disease (Slomovitz et al., 2003). Lymphatic drainage is an important way of tumor dissemination for endometrial cancers (Oz et al., 2014) and histology of UPSC is an independent predictor of postoperative adjuvant treatment in early stage endometrial cancer (Demiral et al., 2014).

Endometrial polyps are outgrows of endometrium; a protrusion to the cavity with a varying size and number. Although there is a debate on the pathogenesis of endometrial polyps, they may contain a hyperplastic foci in $10-25 \%$ of symptomatic cases and may also undergo a malignant transformation in $0.1 \%$ of cases (Savelli et al., 2003). Postmenopausal state, hypertension and obesity are risk factors of malignant transformation in endometrial polyps (Giordano et al., 2007). Unlike endometrioid types; serous uterine tumors are generally seen in non-obese women, arise from an atrophic endometrium without endometrial hyperplasia and they are mostly detected in older women (Bokhman, 1983). By the way our main aim was the evaluation of UPSCs arising from a polyp for any benificial knowledge.

\section{Materials and Methods}

After Institutional Ethics Committee approval at Zekai Tahir Burak Women's Health Education and Research Hospital, we retrospectively evaluated uterine cancers from 2008 to 2014 and 29 out of 570 patients $(5.08 \%)$ were within a serous histologic type. Nine $(31.03 \%)$ of these patients with an uterine papillary serous carcinoma were having a tumor arising from polyp.

Total abdominal hysterectomy, bilateral salpingooophorectomy and pelvic-paraaortic lymphadenectomy with total omentectomy was the standard surgical procedure for all patients. All the operations were performed by the same senior gynecological oncologists.

Individual medical records were analyzed retrospectively from the patient files and computerized database of gynecological oncology unit. Clinical and

${ }^{1}$ Gynecologic Oncology Unit, ${ }^{2}$ Pathology Unit, ${ }^{3}$ Obstetrics and Gynecology, ${ }^{4}$ Department of Obstetrics and Gynecology, Hitit University, Faculty of Medicine, Corum, Turkey and Gynecologic Oncology Unit, Zekai Tahir Burak Women's Health, Education and Research Hospital, Ankara, Turkey*For correspondence: ilkerselcukmd@hotmail.com 
pathological data of patients including age, parity, symptom, tumor marker level, polyp size, myometrial invasion, lymphovascular space invasion (LVSI), stage, pelvic-paraaortic metastasis and peritoneal cytology were reviewed. Staging was determined according to International Federation of Gynecology and Obstetrics clinical staging system, 2009.

Statistical analyses were performed with SPSS for Windows version 17.0 statistical package (SPSS Inc., Chicago, IL, USA). Continuous variables were expressed as mean $\pm \mathrm{SD}$, discrete variables as median (range), and categorical variables as number (percentage). Univariate analysis was performed to reveal the risk factors with $\mathrm{p}<0.05$ set as the cut-off for statistical significance.

\section{Results}

A total of 9 uterine serous carcinoma patients where the tumor arise from a polyp were reviewed. All the patients were postmenopausal. Mean age of patients was $66.7 \pm 8.1$ with a minimum and maximum age of 55 and 79 respectively. Mean parity was $3 \pm 1$. Vaginal bleeding was the most common presenting symptom of patients and pelvic pain was also an additional finding for these patients. Ca-125 level was within normal limits for 6 patients, additionally Ca-15.3, Ca-19.9 and CEA levels were also within normal limits for most of the patients. Mean polyp size was $1.9 \pm 1.3$. Five patients were with a polyp size of $1 \mathrm{~cm}$ or less. One of these patients was with a myometrial invasion of less than $1 / 2$ and one was with greater than $1 / 2$ myometrial invasion. The other three was without myometrial invasion. Two of these patients were having LVSI and one of them was without

Table 1. The Relationship between LVSI, Myometrial Invasion and Polyp Size (NS: Non-Significant)

\begin{tabular}{lccc}
\hline Patient Characteristics & $\begin{array}{c}\text { with } \\
\text { LVSI } \\
(\mathrm{n}=5)\end{array}$ & $\begin{array}{c}\text { without } \\
\text { LVSI } \\
(\mathrm{n}=4)\end{array}$ & p value \\
\hline$>1 / 2$ Myometrial invasion & 3 & 1 & NS \\
$<1 / 2$ Myometrial invasion & 0 & 1 & \\
No myometrial invasion & 2 & 2 & NS \\
Polyp size $>1 \mathrm{~cm}$ & 3 & 1 & \\
Polyp size $\leq 1 \mathrm{~cm}$ & 2 & 3 & \\
\hline
\end{tabular}

myometrial invasion, however the other one was with $>1 / 2$ myometrial invasion. Four patients were with a polyp size of greater than $1 \mathrm{~cm}$. Although three of these patients were with greater than $1 / 2$ myometrial invasion, one of them was without any myometrial involvement. Three of these patients were having LVSI however one of these patients with LVSI was having no myometrial invasion. Predictivity of LVSI according to polyp size and myometrial invasion was not significant $(\mathrm{p}>0.05)$ for UPSC. Additionally polyp size was not related with myometrial invasion for these patients. Table 1 defines the relationship between LVSI, myometrial invasion and polyp size. All the patients who were positive for LVSI were staged higher than $1 \mathrm{~A}$.

We analyzed patients as stage 1A or higher than stage 1A disease (stage II: 1patient, stage IIIC2: 2 patients, stage IVb: 3 patients). Three patients were stage $1 \mathrm{~A}$ and six patients were having a disease higher than stage 1A. Mean Ca-125, Ca-19.9, Ca-15.3 levels were elevated in higher than stage 1A group, however they were within normal limits in stage 1A group. CEA levels were normal in both groups. Mean age and parity were $64.3 \pm 8.1 ; 68.0 \pm 8.6$ and $2.3 \pm 0.5 ; 3.3 \pm 1.0$ respectively in stage $1 \mathrm{~A}$ and higher than stage $1 \mathrm{~A}$ group. Mean polyp size in stage $1 \mathrm{~A}$ and higher than stage $1 \mathrm{~A}$ group was $1.07 \pm 0.11$ and $2.33 \pm 1.5$ respectively.

We did not find a statistical significance for age $(\mathrm{p}=0.439)$, parity $(\mathrm{p}=0.131)$, polyp size $(\mathrm{p}=0.176), \mathrm{Ca}-$ $125(\mathrm{p}=0.121), \mathrm{Ca}-15.3(\mathrm{p}=0.197), \mathrm{Ca}-19.9(\mathrm{p}=0.243)$ and CEA $(\mathrm{p}=0.477)$ levels between two groups. LVSI was positive for 5 patients in >stage 1A group, however in stage 1A group LVSI was not detected for any patient. Lympho-vascular space invasion was showing predictivity for advanced disease $(\mathrm{p}=0.025)$. Greater than $1 / 2$ myometrial invasion was present with 4 patients of $>$ stage 1A group and it was not detected within 2 patients of this group. However $>1 / 2$ myometrial invasion was not predictive for defining an advanced disease $(\mathrm{p}=0.058)$. Malignant peritoneal cytology was detected within 3 patients in >stage $1 \mathrm{~A}$ group however it was not detected in stage $1 \mathrm{~A}$ group. Malignant peritoneal cytology was not meaningful between groups $(\mathrm{p}=0.08)$. Table 2 shows the clinicopathologic features of patients according to the stage.

Table 2. Clinicopathologic Features of Patients According to Stage

\begin{tabular}{lccc}
\hline Patient characteristics & $\begin{array}{c}\text { Stage IA } \\
(\mathrm{n}=3)\end{array}$ & $\begin{array}{c}>\text { Stage IA } \\
(\mathrm{n}=6)\end{array}$ & $\mathrm{p}$ value \\
\hline Age & $64.3 \pm 8.1$ & $68 \pm 8.6$ & 0.439 \\
Parity & $2.3 \pm 0.5$ & $3.3 \pm 1$ & 0.131 \\
Mean Ca-125 (IU/mL) & $10.3 \pm 7.5$ & $253 \pm 230.6$ & 0.121 \\
Mean Ca-19.9 (IU/mL) & $9 \pm 7.5$ & $68 \pm 57$ & 0.243 \\
Mean Ca-15.3 (IU/mL) & $10.6 \pm 9$ & $110.1 \pm 105.4$ & 0.197 \\
Mean CEA (ng/mL) & $1.1 \pm 0.2$ & $1.4 \pm 0.6$ & 0.477 \\
Mean polyp size $(\mathrm{cm})$ & $1.07 \pm 0.1$ & $2.3 \pm 1.5$ & 0.176 \\
LVSI (number=n) & 0 & $5(83.3 \%)$ & 0.025 \\
$>1 / 2$ Myometrial invasion $(\mathrm{n})$ & 0 & $4(66.6 \%)$ & 0.058 \\
Positive peritoneal cytology $(\mathrm{n})$ & 0 & $3(50 \%)$ & 0.08 \\
\hline
\end{tabular}




\section{Discussion}

UPSC is a rare and aggressive form of endometrial cancer with a high risk of metastasis and early recurrences even without myometrial invasion. In endometrioid endometrial cancer adjuvant theraphy is primarily based on uterine risk factors (Nugent et al., 2012), however in UPSC there is no valid methodology for risk definition. Moreover histological and genomic studies have distinguished UPSC from endometrioid endometrial cancer and pointed similarities to serous ovarian cancer (Desai et al., 2013).

Endometrial polyps are generally seen in perimenopausal women. They combine focal stromal development and vessel structure. Additionally hyperplastic foci and malignant transformation could be seen in polyps. UPSC is a distinct form of endometrial cancer unlike endometrioid carcinoma which arises from hyperplastic endometrium by the effect of estrogen, UPSCs are related to atrophic endometrium without estrogen stimulation and have different patterns of spread from endometrioid carcinoma (Idrees et al., 2013).

Silva and Jenkin (Silva and Jenkins, 1990) reported 16 uterine serous carcinoma patients involving an endometrial polyp. They reported a high rate of extrauterine disease or extrauterine recurrences independent from myometrial or lymphovascular invasion. Having a stage 1 disease without additional risk factors was not protective from extrauterine recurrences and they claimed serous carcinomas involving endometrial polyps as an aspect of multicentric disease; in the pelvis and abdominal surfaces. In endometrioid carcinoma myometrial invasion is a powerful predictor of extrauteine spread however in serous carcinomas this is not notable and grading the tumor does not provide prognostic significance because all the tumors are in a high grade histologic nature (del Carmen et al., 2012). Carcangiu et al. (1997) reported two deaths out of 13 stage $1 \mathrm{~A}$ uterine serous carcinoma patients, these two were with an endometrial polyp; tumor was invading both endometrium and polyp thus a multifocal tumor growth should not be underestimated for uterine serous carcinomas. Sherman et al. (1992) also stated the risk of a widespread metastasis, recurrence and death even in patients where myometrial invasion is less than $1 \mathrm{~mm}$ or where the tumor is confined to polyps. On the other hand it was also cited that deep myometrial invasion is an important predictor of lymph node metastasis (Sethasathien et al., 2014). Hendrickson et al. (1982) showed upper abdomen and abdominal cavity as a side of recurrences and pointed at the similarity between UPSC and serous ovarian carcinomas for spreading over peritoneal surfaces.

Two of our patients $(50 \%, n=2$ of 4$)$ who were without any myometrial invasion were having an abdominal disease. It should be noted that they were with LVSI. Three patients who were having paraaortic lymph node metastasis $(66.6 \%)$ were also with LVSI. One of these patients was having a polyp size lesser than $1 \mathrm{~cm}$ and myometrial invasion was also less than $1 / 2$ for that patient. Cirisano et al. (1999) found $13 \%$ of patients with paraaortic lymph node metastasis in the absence of myometrial invasion. Slomovitz et al. (2003) found lymph node involvement $19 \%$ in patients without myometrial invasion. They found stage, lymph node status, LVSI and depth of myometrial invasion as prognosticators of a poor disease. In that manner complete surgical staging and debulking of all tumor sides may improve survival analysis.

We found only LVSI significant for advanced disease. However Geisler et al. (1999) found myometrial invasion as an indicator of advanced disease. Slomovitz et al. (2003) found LVSI as a factor of advanced stage and extra-uterine dissemination. Vascular and lymphatic invasion is a strong finding of uterine serous tumors with a high detection rate which makes extrauterine spread, recurrence and death easier in the absence of myometrial invasion (Sherman et al., 1992; Goff et al., 1994; Wheeler et al., 2000). Even for patients with a minimal uterine serous tumor which defines a disease limited to endometrium, the risk of extrauterine disease is $45 \%$ (Wheeler et al., 2000). In these conditions especially LVSI shows predictivity than other uterine factors. We know stage as the primary prognostic determiner meanwhile recurrences and risk of metastasis increase with advanced stage (Rauh-Hain et al., 2010). In that manner LVSI should also be an important finding for the prediction of advanced disease.

Having an advanced disease shorten patients' survival in serous uterine tumors. LVSI is a good prognosticator and significantly predicts an advanced disease for patients with or without any myometrial invasion.

\section{References}

Bokhman JV (1983). Two pathogenetic types of endometrial carcinoma. Gynecol Oncol, 15, 10-7.

Carcangiu ML, Tan LK, Chambers JT (1997). Stage IA uterine serous carcinoma: a study of 13 cases. Am J Surg Pathol, 21, 1507-14.

Cirisano FD, Jr., Robboy SJ, Dodge RK, et al (1999). Epidemiologic and surgicopathologic findings of papillary serous and clear cell endometrial cancers when compared to endometrioid carcinoma. Gynecol Oncol, 74, 385-94.

del Carmen MG, Birrer M, Schorge JO (2012). Uterine papillary serous cancer: a review of the literature. Gynecol Oncol, 127, 651-61.

Demiral S, Beyzadeoglu M, Sager O, et al (2014). Evaluation of treatment outcomes of early-stage endometrial cancer radiotherapy: a single center experience. Asian Pac J Cancer Prev, 15, 9599-602.

Desai NB, Kiess AP, Kollmeier MA, et al (2013). Patterns of relapse in stage I-II uterine papillary serous carcinoma treated with adjuvant intravaginal radiation (IVRT) with or without chemotherapy. Gynecol Oncol, 131, 604-8.

Geisler JP, Geisler HE, Melton ME, et al (1999). What staging surgery should be performed on patients with uterine papillary serous carcinoma? Gynecol Oncol, 74, 465-7.

Giordano G, Gnetti L, Merisio C, et al (2007). Postmenopausal status, hypertension and obesity as risk factors for malignant transformation in endometrial polyps. Maturitas, 56, 190-7.

Goff BA, Kato D, Schmidt RA, et al (1994). Uterine papillary serous carcinoma: patterns of metastatic spread. Gynecol Oncol, 54, 264-8.

Hendrickson M, Ross J, Eifel P, et al (1982). Uterine papillary serous carcinoma: a highly malignant form of endometrial adenocarcinoma. Am J Surg Pathol, 6, 93-108. 


\section{Selcuk Ilker et al}

Huh WK, Powell M, Leath CA, et al (2003). Uterine papillary serous carcinoma: comparisons of outcomes in surgical Stage I patients with and without adjuvant therapy. Gynecol Oncol, 91, 470-5.

Idrees R, Din NU, Fatima S, et al (2013). Serous carcinoma arising in endometrial polyps: clinicopathologic study of 4 cases. Ann Diagn Pathol, 17, 256-8.

Moore KN, Fader AN (2011). Uterine papillary serous carcinoma. Clin Obstet Gynecol, 54, 278-91.

Nugent EK, Bishop EA, Mathews CA, et al (2012). Do uterine risk factors or lymph node metastasis more significantly affect recurrence in patients with endometrioid adenocarcinoma? Gynecol Oncol, 125, 94-8.

Oz M, Ozgu E, Korkmaz E, et al (2014). Utility of frozen section pathology with endometrial pre-malignant lesions. Asian Pac J Cancer Prev, 15, 6053-7.

Rauh-Hain JA, Growdon WB, Schorge JO, et al (2010). Prognostic determinants in patients with stage IIIC and IV uterine papillary serous carcinoma. Gynecol Oncol, 119, 299-304.

Savelli L, De Iaco P, Santini D, et al (2003). Histopathologic features and risk factors for benignity, hyperplasia, and cancer in endometrial polyps. Am J Obstet Gynecol, 188, 927-31.

Sethasathien P, Charoenkwan K, Siriaunkgul S (2014). Accuracy of intraoperative gross examination of myometrial invasion in stage I-II endometrial cancer. Asian Pac J Cancer Prev, 15, 7061-4.

Sherman ME, Bitterman P, Rosenshein NB, et al (1992). Uterine serous carcinoma. A morphologically diverse neoplasm with unifying clinicopathologic features. Am J Surg Pathol, 16, 600-10.

Siegel R, Naishadham D, Jemal A (2013). Cancer statistics, 2013. CA Cancer J Clin, 63, 11-30.

Silva EG, Jenkins R (1990). Serous carcinoma in endometrial polyps. Mod Pathol, 3, 120-8.

Slomovitz BM, Burke TW, Eifel PJ, et al (2003). Uterine papillary serous carcinoma (UPSC): a single institution review of 129 cases. Gynecol Oncol, 91, 463-9.

Wheeler DT, Bell KA, Kurman RJ, et al (2000). Minimal uterine serous carcinoma: diagnosis and clinicopathologic correlation. Am J Surg Pathol, 24, 797-806. 